\title{
Interoperability in Nigeria Healthcare System: The Ways Forward
}

\author{
Iroju Olaronke \\ Department of Computer Science, Adeyemi College of Education, Ondo, Nigeria.
}

Email: irojuolaronke@gmail.com

Gambo Ishaya

Department of Computer Science and Engineering, Obafemi Awolowo University, Ile-Ife, Nigeria

Email:ipgambo@gmail.com@gmail.com

Ikono Rhoda

Department of Computer Science and Engineering, Obafemi Awolowo University, Ile-Ife, Nigeria.

E-mail: rhoda_u@yahoo.com

Olaleke Janet

Department of Computer Science, Adeyemi College of Education, Ondo, Nigeria.

Email: shollyjane@yahoo.com

\begin{abstract}
Interoperability of health related information is one of the agendas of many counties in the world, with no exception to Nigeria and other developing countries. This is because healthcare costs are rising exponentially. However, interoperability of health related information seem largely unattainable in Nigeria due to reluctance to change from the traditional paper based healthcare system to the use of e-health systems, inadequate ICT infrastructure, poor utilization of the available ICT resources, erratic power supply, increased burden of underdevelopment, poverty, political instability, shortage of educational capacity in Nigeria rural and urban healthcare centers, low level of ICT awareness, poor maintenance culture as well as corruption. Consequently, the healthcare system in Nigeria is saddled with high cost, high rate of disease outbreak driven by HIV/AIDs, malaria and other infectious diseases which results in a high rate of mortality. Nevertheless, the urgent need to meaningfully exchange safe and reliable health information is a key priority to the healthcare system in Nigeria as the qualities of patients' care depend majorly on the timely acquisition, processing and retrieval of data related to the patient. Thus, this paper attempts to unravel the factors hindering interoperability in the Nigeria healthcare system and suggests ways of making total interoperability a reality in Nigeria healthcare system as well as other developing countries.
\end{abstract}

Index Terms - Nigeria healthcare system, ICT, interoperability.

\section{INTRODUCTION}

The Nigeria healthcare system is implemented and maintained at various clinics and hospitals owned by the three tiers of government which include the local, state and the federal government. The need for healthcare providers in the various hospitals to interact and exchange patients' information is essential for the continuity of patients' care. Interoperability in the Nigeria healthcare system is a challenge to healthcare practitioners because the seamless and meaningful exchange of health related information relies heavily on the use of Information and Communication Technology (ICT) tools which are used to make better decisions at the point of care and thus to deliver high-quality of care to patients. Nevertheless, the process of achieving a meaningful exchange of patient information in Nigeria healthcare system is plagued with the limited use of ICT facilities ${ }^{[1][2]}$. Furthermore, the limited ICT tools used in the Nigeria healthcare delivery system are developed independently by diverse software and hardware vendors with different methods, procedures and processes, which results in autonomous and heterogeneous healthcare systems. Thus, the processing and retrieval of health information from disparate electronic health information systems at the point of care becomes a big issue. Consequently, the healthcare delivery system in Nigeria is inadequate, characterized by increased cost and increased inaccessibility to healthcare especially for those in remote rural areas as well as a high rate of mortality. Hence, the deployment of an adequate and interoperable information and communication technology tools for accurate, meaningful, secured, and cost-effective information exchange in Nigeria healthcare delivery system is very imperative. This paper therefore attempts to unravel the poor state of interoperability in Nigeria healthcare system. It also examines the factors affecting the seamless and meaningful exchange of information in Nigeria healthcare system. This paper also suggests ways of making complete interoperability a reality in Nigeria healthcare system. 
The remainder of this paper is organized as follows: Section 2 examines different views of interoperability in healthcare. Section 3 describes the Nigeria healthcare system. Section 4 analyzes the barriers to interoperability in the Nigeria healthcare system. Section 5 describes the consequences of lack of interoperability to Nigeria healthcare while Section 6 suggests ways of realizing complete interoperability in Nigeria healthcare system. This was followed by the conclusion in Section 6.

\section{DIFFERENT VIEWS OF INTEROPERABILITY IN HEALTHCARE}

The term interoperability has been extensively defined by different authors, however, with the same goal which is the creation of better health for individuals, communities, nations and the world at a lowered cost. A widely used definition of interoperability by the Institute of Electrical and Electronics Engineers (IEEE) Standard Computer Dictionary [3] is the ability of two or more systems or components to exchange information and to use the information that has been exchanged. The National Alliance for Health Information Technology [4] also defined interoperability as the ability of different information technology systems and software applications to communicate, exchange data accurately, effectively, and consistently, and to use the information that has been exchanged. In addition, interoperability as defined by the Executive Order signed by President Bush of the United States of America in August 2006 is the ability to communicate and exchange data accurately, effectively, securely, and consistently with different information technology systems, software applications, and networks in various settings, and exchange data such that clinical or operational purpose and meaning of the data are preserved and unaltered [5]. Furthermore, Yum and Drogemuller [6] viewed interoperability as the ability of diverse computer programs to exchange/share information without any loss of content or meaning. From the definitions above, it can be deduced that interoperability is majorly concerned with the seamless exchange of information, and the preservation of the meaning of the information exchanged by communicating systems so that it can be effectively used by the receiving system.

There are different levels of interoperability in healthcare as identified by different authors. For instance, the National Alliance for Health Information Technology [3] identified four basic levels of interoperability in healthcare. These levels include the exchange of data in non- electronic formats such as the use of paper based system which is albeit a highly constrained form of interoperability, the exchange of data in electronic formats such as data exchange via electronic mail, the use of data that machines can organize such as labeled images or documents and data interpretation by machines. From the critical evaluation of these levels of interoperability, it can be inferred that the levels of interoperability by the National Alliance for
Health Information Technology [3] is categorized into two basic levels, that is, the exchange of data (technical or syntactic interoperability), as well as the interpretation and the use of data (semantic interoperability). The National Alliance for Health Information Technology levels of interoperability is summarized in Fig.1.

\begin{tabular}{l} 
Semantic Interope rability \\
a. Data Interpre tation \\
b. Data Use \\
\hline \multicolumn{1}{|c|}{ Technical Inte roperability } \\
a. Data exchange in non-electronic \\
format \\
b. Data exchange in electronic format \\
\end{tabular}

Figure 1: A View of the National Alliance for Health Information Technology (2005) levels of Interoperability in healthcare

On the other hand, the Health Level 7 (HL7) Interoperability Workgroup classified interoperability in healthcare into three basic levels. This includes technical interoperability, semantic interoperability and process interoperability which is often omitted at various levels of healthcare interoperability [5]. The HL7 Interoperability Workgroup referred to technical interoperability as the transmission and reception of information that can be used by a person but which cannot be further processed into semantic equivalents by software. Thus, technical interoperability has to do with the conveyance of data and not on semantics. Its basic goal is to reduce the effect of distance in healthcare. The HL7 Interoperability Workgroup described semantic interoperability as the ability of information shared by systems to be understood so that data can be processed by the receiving system [5]. Dolin and Alschuler [7] however elaborated the definition of semantic interoperability as the ability to import utterances from another computer without prior negotiation and still having the decision support system, data queries and business rules work reliably against these utterances. Process interoperability as also defined by HL7 interoperability workgroup is an emerging concept that has been identified as a requirement for successful system implementation into actual work settings by improving safety and quality in healthcare settings [5]. Process interoperability includes the methods necessary for the optimal integration of computer systems into actual work settings. It ensures seamless communication between different healthcare systems by developing shared understanding of their process artifacts [8]. These three levels of interoperability are interdependent, and all three are needed to deliver significant healthcare benefits. The HL7 Interoperability Workgroup levels of interoperability are summarized in Fig. 2. 


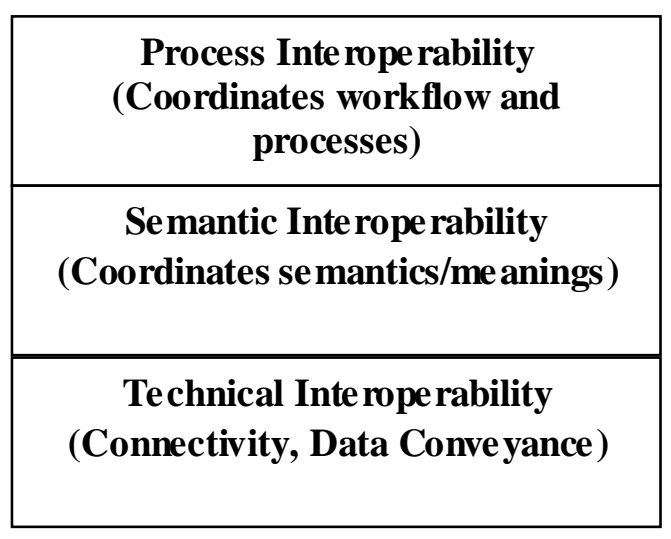

Figure 2: A View of HL7 Interoperability Workgroup Levels of Interoperability in healthcare

This study therefore examines the state of interoperability in Nigeria healthcare delivery system in the light of the HL7 interoperability workgroup levels of interoperability. This is because it considers the two widely emphasized levels of interoperability in healthcare (technical/syntactic and semantic interoperability) in addition to process interoperability. Moreover, in order to achieve complete interoperability in healthcare, all aspects of interoperability must be taken into consideration [9]. Furthermore, the more these three levels of interoperability are comprehended, the less likely it is to underestimate the efforts required to make Nigeria healthcare delivery system interoperable. Consequently, the quality of healthcare will be improved, healthcare cost will be reduced, accessibility to health information even in the remotest part of the rural areas would increase and patients' satisfaction will be achieved in Nigeria healthcare system.

\section{THE NIGERIA HEALTHCARE SYSTEM}

The healthcare delivery system in Nigeria is composed of a three tier structure which is owned, implemented and maintained at the federal, state and local government levels [10]. However, the mixed nature of Nigeria's economy granted private care providers a role to play in the healthcare delivery system of Nigeria. According to Federal Ministry of Health [11], the federal government of Nigeria/ the federal ministry of health is responsible for tertiary healthcare which comprises of Teaching and Specialist hospitals as well as the federal medical centers. The federal government is also responsible for setting the overall healthcare policies and providing technical support to the healthcare system. The state government is responsible for secondary healthcare which is essentially the general hospitals. The state government is also responsible for the regulation and provision of technical support for primary healthcare, while the local government is responsible for primary healthcare which is organized through the wards, health districts and health centers. According to Oluwagbemi and
Achimugu [12], the local communities form the heart of the primary healthcare.

The Nigeria healthcare system consists of enormous silos of paper-based systems that are frag mented and this result in proprietary control over health information across the nation. In addition, the healthcare system also consist of electronic data which are stored in numerous and widely scattered repositories which leads to the fragmentation of health records, thus exposing the healthcare system to risk of infraction. Hence, the various hospitals and clinics function independently and their interconnectivity and interoperability have continued to be a big challenge because the access to health information for prompt and efficient decision making in disparate health information systems is difficult. Consequently, the Nigeria healthcare system is in a woeful state. Hence, Nigeria's overall health system performance was ranked 187th position among 191 Member States by the World Health Organization in year 2000 [13]. The Nigeria healthcare system is depicted in a chart in Fig. 3. 


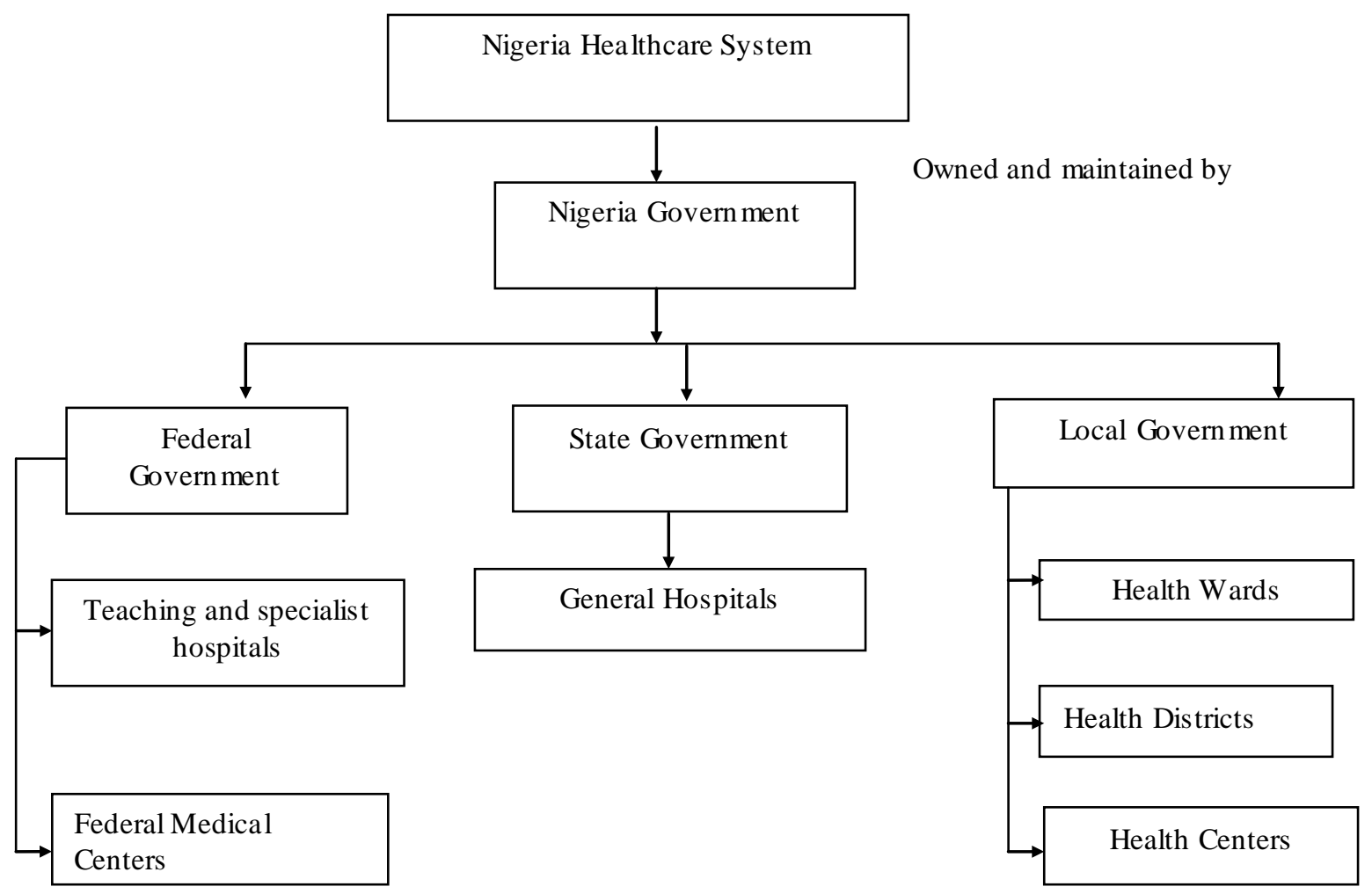

Figure 3: A Chart depicting Nigeria Healthcare System

\section{BARRIERS TO INTEROPERABILITY IN NIGERIA HEA LTHCA RE SYSTEM}

The lack of interoperability in healthcare systems and services has long been identified as one of the major challenges in healthcare [14]. Thus, this section appraises the factors impeding technical, semantic and process interoperability in Nigeria healthcare.

\subsection{Factors Hindering Technical Interoperability in Nigeria Healthcare System}

The factors impeding technical interoperability, that is, the seamless exchange of health information in Nigeria healthcare system include:

1) Inadequate ICT Facilities: In recent times, Information and Communication Technology tools such as computer hardware, software and bandwidth have been fully deployed in major sectors in Nigeria such as banking, manufacturing and the oil and gas industries. However, the health sector is yet to fully witness the use of these facilities especially in rural hospitals or healthcare centers where the non-availability of medical information and physical barriers prevent patients from interacting with healthcare providers for effective healthcare services ${ }^{[15]}$. For instance, only about $4 \%$ of General Practitioners in Nigeria use Information Technology (IT) applications for clinical purposes ${ }^{[12]}$. This has soared medical diagnosis and treatment of Nigerians overseas. For instance it was reported that Nigerians spend about $\$ 78$ billion annually on medical tourism overseas in India, Singapore, South Africa and even Ghana [16]. Moreover, the healthcare industry is characterized by the extensive use of information which needs to be seamlessly exchanged amongst disparate healthcare providers especially when distance is a critical factor. Nonetheless, the interoperability of health related information in Nigeria healthcare remains a challenge as a result of the inadequacy of ICT facilities in the health sector.

2) Erratic Power Supply: Most ICT facilities are driven by electricity. The Nigeria government has not been able to provide uninterrupted power supply to its citizens. Idowu et al. [1] identified that Nigeria rarely has stable and reliable electricity for ten consecutive hours in a day. However, the effective use of ICT facilities requires stable, reliable and regular power supply. Consequently, the effective deployment of the available ICT facilities or e-health systems for the seamless exchange of health information amongst diverse healthcare professionals is a formidable challenge in the Nigeria healthcare system. For instance, Akinyede et al. [15] reported that one of the major impediments to the implementation of telemedic ine in Nigeria is epileptic power supply.

3) Reluctance to Change: The Nigeria healthcare sector at present rely largely on paper based system, 
unlike other sectors like the banking sector and the oil and gas industry. The paper based system is inherently erro r-prone because healthcare providers may not be able to communicate effectively during patents care; this however leads to medical errors. Despite the demerit of the paper based system, Nigeria healthcare practitioners are unwilling to switch to new technological developments due to the fear of job loss, inadequate administrative support for IT and related practice changes, lack of trust and other legal concerns with respect to joint IT solutions, lack of necessary and appropriate skills for the operation of the ICT tools as most health professionals have a low level of computer literacy, as well as privacy and security issues as patients information are meant to be confidential. Hence, the use of ICT tools for the seamless exchange of health data seems unattainable in the context of Nigeria healthcare system, despite that it is one of the objectives of the Millennium Development Goals to ensure that the use of ICT solutions provide easy access to health information across geographical boundaries at the three levels of healthcare [17].

4) Proliferation of Incompatible E-Health Systems: Most e-health systems used in Nigeria healthcare systems today are proprietary such as the Made in Nigeria Primary Healthcare and Hospital Information System (MINPHIS), a commercial software developed in the late 1990s by the collaborative efforts of Finnish research team of the University of Kuopio, Department of Computer Science and Engineering, Obafemi Awolowo University and Obafemi Awolowo University Teaching Hospital. These systems are usually developed by diverse hardware/software developers at different geographical locations and with different tools, methods, processes and procedures. This results in a large number of noninteroperable, incompatible, heterogeneous and distributed proprietary health systems for the storage, processing and retrieval of health related information. This however strengthens information silos in healthcare, and thus the seamless exchange of information as well as the integration of health information in disparate electronic health information system becomes a challenge.

5) Corruption: Corruption in Nigeria is a cankerworm that has eaten deep into the fabrics of the country, and thus negatively affected all the sectors of the country including the healthcare sector. For instance, between 1996 and 2006, Nigeria was ranked the most corrupt and the second most corrupt nation in the world respectively. Thus Nigerians are often associated with fraud and corruption [18]. This factor has undoubtedly adversely affected the healthcare system as most funds allocated to the purchase and maintenance of ICT facilities for seamless health information exchange and interoperability is often misappropriated.

6) Security: Patients' information are meant to be confidential, however the healthcare industry is plagued with fraud and insecurity. Thus, the risk of security problems such as unauthorized access to patients' information through ICT facilities by malicious individuals is one of the factors affecting interoperability in healthcare.

4.2 Factors Hindering Semantic Interoperability in Nigeria Healthcare System

Having highlighted the obstacles of technical interoperability in Nigeria healthcare system in the previous section, this section examines the factors hindering the successful achievement of semantic interoperability in Nigeria healthcare system. However, these challenges are not limited to Nigeria healthcare system alone but also other healthcare systems in both developed and developing nations.

1) Rapid Growth of Incompatible Healthcare Vocabularies/Ontologies: Vocabularies are controlled collections of concepts that capture med ical knowledge from a particular perspective or point of view [19]. They serve as dictionaries that organize and describe words and related concepts and they also facilitate information capture, storage, exchange, and retrieval (e.g. International Classification of Diseases 9). The main purpose of vocabularies/ontologies is to ensure semantic interoperability[20]. However, since healthcare vocabularies are developed in the context of various assumptions and targeted for multiple uses, there is usually the possibility of having multiple expressions referring to the same concept [19]. This however leads to conflict, ambiguity and confusion whenever information are integrated or exchanged amongst healthcare professionals. Nonetheless, the number of termino logical systems with mutually incompatible definition is growing exponentially day by day [21]. This wide explosion of incompatible healthcare vocabularies has extensively contributed to lack of semantic interoperability in healthcare.

2) Lack of a Unified Healthcare Standard: Interoperability of healthcare systems depends on the use of recognized standards which describes the technical specifications, methods and processes. However, the Nigeria healthcare system like other healthcare systems in most part of the world lacks a common standard. For instance, there are still no satisfactory international standards for name, date and address. In the case of date, some healthcare systems use the format $\mathrm{dd} / \mathrm{mm} / \mathrm{yy}$ while others use $\mathrm{mm} / \mathrm{dd} / \mathrm{yy}$. This often leads to misunderstanding and subsequently medical errors

3) Use of non-standardized abbreviations in medical texts: The healthcare system is made up of clinical reports which are compact and contain 
abbreviations. For instance, "pe”, may mean physical examination, pleural effusion, or pulmonary embolism. Hence, a major problem with abbreviations is that they are highly ambiguous [22]. This problem presents a major challenge to semantic interoperability in the healthcare domain because it can result to gross medical errors, and thus wrong medical diagnostics and treatment.

4) Lack of a Unified Terminology Set in Healthcare: The healthcare system generally lacks a unified terminology set and as such different terminologies can be used to represent the same concept. For instance the word cardiac and heart represent the same concept; also myocardial infarction and heart attack are synonymous terms. In addition, a term can be used to represent different concepts. For instance when the term mass occurs in a radiological report of the chest, it means mass in lung, whereas if it occurs in a mammography report, it means mass in breast [22]. This however poses a great challenge to computer based healthcare systems when terms are not explicitly defined. This is because it is often difficult for computer system to capture the semantics of information [23].

4.3 Factors Hindering Process Interoperability in Nigeria Healthcare System

The factors hindering process interoperability in Nigeria healthcare include the following:

1) Workflow Heterogeneity: Most hospitals in Nigeria are characterized by heterogeneous workflows which involve numerous tasks such as data acquisition, storage, processing, retrieval and information flow. Thus, the processes involved in data acquisition, storage, processing and retrieval differ in various hospitals and clinics. This however affects process interoperability if the interoperating health organizations do not understand the intricacies and heterogeneity in the workflows.

\section{CONSEQUENCES OF LACK OF INTEROPERA BILITY IN NIGERIA HEA LTHCARE SYSTEM}

This section carefully and critically examines the effects of lack of complete interoperability in the Nigeria healthcare delivery system.

1) High Rate of Disease Outbreak: Nigeria is a country that is saddled with a high rate of disease outbreak such as malaria, tuberculosis and Human Immunodeficiency Virus/Acquired Immunodeficiency Syndrome (HIV/AIDS). For instance, Arikpo et al. [24] reported that about 3.6 million of Nigeria population are HIV positive or have developed AIDS which is equivalent to a prevalence of $5.4 \%$ of the adult population. In addition, Arikpo et al. [24] also stated that more than 300,000 individuals in Nigeria die from AIDS annually. One of the reasons for this outbreak is because Nigeria citizens' awareness to health knowledge is low [13]. This is because the use of ICT facilities to facilitate the promotion of the awareness of these diseases, and the necessary precautionary measures that are needed to prevent disease outbreak is low. Thus, it becomes difficult for individuals to improve their lifestyle to avoid these diseases.

2) High rate of mortality: Nigeria as a nation is characterized by high rate of mortality which is due to lack of interoperability in the healthcare system. For instance, about 52,900 women and 250,000 new born babies die annually [25]. This however is usually caused by sheer lack of interoperable ICT based healthcare platforms which could have facilitated effective data sharing and communication amongst the care givers and their patients irrespective of their geographical locations.

3) Increased healthcare cost: The lack of ICT tools and technologies for the facilitation of effective sharing and communication of data, information, and knowledge among various stakeholders in the circle of patient care has led to an increased healthcare cost in Nigeria. Furthermore, most rural communities in Nigeria are inaccessible due to poor transportation system. Thus, the access to quality healthcare service in these regions becomes a formidable challenge, as the rural dwellers have to travel a long distance to the urban areas at a high cost for quality healthcare. Also, the lack of interoperable ICT tools has further widened the healthcare service delivery gap between the urban and rural populace of Nigeria because patients from the rural centers get quality healthcare at a high cost by leaving their cities. Thus, most rural dwellers in the local communities find it impossible to have access to healthcare due to the increased cost of healthcare.

4) Increased inaccessibility to health information at the point of care: Information is a very vital component for decision making in the healthcare sector, and thus needs to be effectively communicated amongst healthcare providers and their patients. However, the lack of interoperability in Nigeria healthcare system has led to broken health records and fragmentation of patients' information. Consequently, it becomes very difficult to access as well as present a clear and complete picture of a patient at the point of care.

5) Problems of data integration: The Nigeria healthcare system is composed of heterogeneous proprietary systems which are developed by diverse individuals on different hardware/software platforms with diverse tools, technologies, methods, processes and procedures according to their needs and beliefs. Consequently, this lack of 
interoperability makes data integration from the heterogeneous system a difficulty.

\section{MITIGATION APPROA CHES}

The consequences of lack of complete interoperability in Nigeria healthcare delivery system are severe. This is because of the critical nature of healthcare, as it deals directly with human lives. Consequently, this section suggests ways of making complete interoperability a reality in the Nigeria healthcare delivery system.

1) Provision of adequate and interoperable ICT tools for healthcare service and delivery: The Nigeria government should provide adequate, compatible and interoperable ICT tools such as computers, internet facilities, Personal Digital Assistant (PDA), telephones (Landlines and Global System for Mobile Communication (GSM)) and radio links at the three tiers of healthcare. This will facilitate the real time access to health -related information any time and at diverse geographical locations especially when distance is a barrier. This will inevitably bridge the digital divide between the rural and urban communities, thereby saving lives, healthcare cost and time.

2) Education strategy: Nigeria educational curriculum planners and developers should integrate ICT related courses such as introduction to health informatics, electronic health systems, ICT for development, as well as systems analysis and design at the primary, secondary and tertiary levels of education, so that both the urban and rural dwellers will have the full knowledge of the relevance of interoperability to healthcare.

3) Adoption of a unified standard for the healthcare industry: The federal government of Nigeria should standardize the healthcare system for privacy, security, data storage, retrieval and exchange. A common classification system should be enforced in the healthcare system to resolve the ambiguous use of medical terms and semantic heterogeneity. Alternatively, the use of an ontology matching system should be encouraged in the Nigeria healthcare system. This will facilitate the easy identification of medical terms that are semantically related. The process of ontology matching will also enable healthcare practitioners to identify similar terms that are represented heterogeneously, thereby facilitating the interpretation of medical terms

4) Provision of adequate power supply: The federal government should provide stable and constant electricity supply for the uninterrupted functioning of ICT tools in healthcare. This will facilitate the seamless and uninterrupted exchange of information amongst healthcare systems.

5) Provision of an electronic Health legislative framework: The legislative arm of the government should pass a bill that will support the use of electronic health systems in Nigeria healthcare. The government should also provide an enabling environment for the successful implementation, maintenance and sustenance of e-health in Nigeria. This will result in efficient and cost effective healthcare services at the three tiers of healthcare.

\section{CONCLUSION}

Interoperability is crucial to the healthcare system because it facilitates the easy access to health-related information amongst disparate healthcare providers. However, interoperability in the Nigeria healthcare system is peculiar and seems a mirage because of the formidable challenges that are associated with it. Thus, healthcare cost, disease outbreak, mortality rate and inaccessibility to patients' information at the point of care are increasing rapidly in the Nigeria healthcare system. Consequently, this paper examined interoperability in the context of Nigeria healthcare by revealing the obstacles to the seamless exchange of meaningful health information in the Nigeria healthcare system. The study also analyzed the consequences of lack of interoperability in the Nigeria healthcare system and presents the strategies for achieving complete interoperability in the Nigeria healthcare system.

\section{REFERENCES}

[1] Idowu P, Cornford D, et al. Health informatics deployment in Nigeria [J]. Journal of Health Informatics in Developing Countries, 2008, 2:1523.

[2] Ayodele, C.B. Hospital information systems in Nigeria: A review of literature [J]. Journal of Global Healthcare Systems, 2011, 1(3):1-26.

[3] Institute of Electrical and Electronics Engineers Standard Computer Dictionary. A Compilation of IEEE Standard Computer Glossaries, Institute of Electrical and Electronics Engineers, USA, 1990.

[4] Heubusch K. Interoperability: What it means, why it matters [J]. Journal of AHIMA, 2006, 77(1):2630.

[5] Gibbons P, Arzt N, et al. Stanford J. Coming to terms:scoping interoperability for healthcare[R]. United States of America: Health Level Seven International, 2007.

[6] Yum K.K, and Drogemuller R. How much interoperability can be achieved for the construction industry today?[A]. Hong Kong: INCITE World IT for design and construction, 2002.

[7] Dolin R, Alschuler L. Approaching semantic interoperability in Health level seven [J]. Journal of American Medical Information Association, 2011, 18:99-103.

[8] Khan W A, Hussain M, Latif K,et al. Process interoperability in healthcare systems with 
dynamic semantic web services[A]. SpringerVerlag Wien, 2013.

[9] Health Governance Initiative. On Semantic and Technical Interoperability[A]. United States of America: eHealth Governance Initiative, 2012.

[10] Rais A. Health Care Patterns and Planning in Developing Countries [A]. London: Greenwood Press, 1991.

[11] Federal Ministry of Health. The National health policy and strategy to achieve health for all Nigerians. Nigeria: Federal Ministry of Health, 1998.

[12] Oluwagbemi $O$, and Achimugu P. Lack of integration in software systems for health practitioners in Nigeria: The way forward [J]. Journal of Health Informatics in Developing Countries, 4(1): 47-51.

[13] Odusote A. O. ICT for Public Health Care Delivery in Nigeria: Challenges, Opportunities and Milestones[C]. In: Proceedings of e-Nigeria Conference, Abuja, Nigeria, 2010, 1-5.

[14] Iroju O, Soriyan A, et al. Interoperability in Healthcare: Benefits, challenges and resolutions[J]. International Journal of Innovation and Applied Studies, 2013, 3(1): 262-270.

[15] Akinyede R. O, Daramola O. A, et al. Towards delivering high quality healthcare through an integrated national health information system and telemedicine development, problems and recommended solutions [J]. Canadian Journal on Computing in Mathematics, Natural Sciences, Engineering and Medicine, 2012, 3(6):180-187.

[16] Business Day Newspaper. Absence of national eHealth strategy, threat to nation's healthcare delivery [A].Nigeria: Business Day, 2012.

[17] Dzidonu C. An Analysis of the Role of ICTS to achieving the MDGS [R]. United States of America: Division for Public Administration and Development Management, United Nations Department of Economic and Social Affairs (UNDESA), 2010.

[18] Ayodele C. B. Hospital information systems in Nigeria: A review of literature $[\mathrm{J}]$. Journal of Global Healthcare Systems, 2011, 1(3): 1-26.

[19] Bock C, Carnahan L, et al. Healthcare strategic focus area: clinical in formatics [R]. United States of A merica: National Institute of Standards and Technology, 2005.

[20] Shvaiko P.Iterative Schema-Based semantic matching”, Trento: International Doctorate School, Information and Communication Technology Department, University of Trento, 2006.

[21] Lenz R, Beyer M, et al. Semantic integration in healthcare networks $[\mathrm{J}]$. Connecting Medical Informatics and Bio-Informatics, 2005, 385-390.

[22] Friedman C. Semantic text parsing for patient records [A]. Department of Biomedical Informatics [A], New York: Columbia University Press, 2005.
[23] Bittner T, Donnelly M, et al. Ontology and semantic interoperability. Institute for Formal Ontology and Medical Information Science (IFOMIS). Saarland: Saarland University, 2006.

[24] Arikpo A, Etor R, et al. Development imperatives for the twenty-first century in Nigeria [J]. Journal of Convergence, 2007, 40(1): 55-66.

[25] Nwogbo K. Underpinning Health Care with ICT [A]. Nigeria: Nigeria Communication Week, 2005.

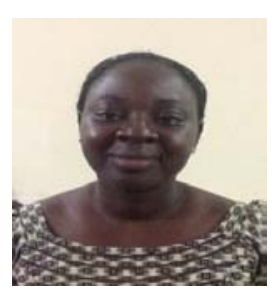

Iroju Olaronke: has a B.Sc. in Computer Technology at Babcock University, Nigeria. She also has M.Sc and $\mathrm{PhD}$ in Computer Science at Obafemi Awolowo University, Nigeria. She is a lecturer at the Department of Computer Science, Adeyemi College of Education, Ondo, Nigeria. Her research interest is on health informatics, interoperability and ontology matching.

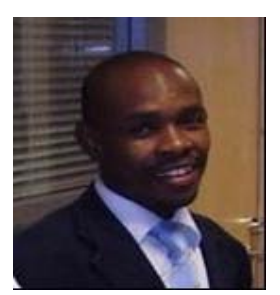

Gambo Ishaya: is a lecturer of Computer Science at the Obafemi Awolowo University Ile-Ife, Nigeria. $\mathrm{He}$ is pursuing a $\mathrm{PhD}$ degree in Computer Science. He has got a good number of publications in reputable journals and learned conferences. His research interest is in Information systems design and methodology, software engineering with emphasis on Software Architecture and software quality issues.

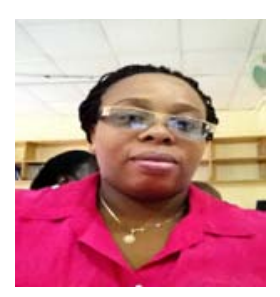

Ikono Rhoda: is a lecturer of Computer Science at the Obafemi Awolowo University Ile-Ife, Nigeria. She has a $\mathrm{PhD}$ in Computer Science at Obafemi Awolowo University, Nigeria. Her research interest is in Information System, Health Informatics, and Software Product Usability.

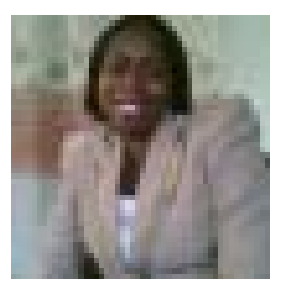

Olaleke Janet: She is a lecturer at the Department of Computer Science, Adeyemi College of Education, Ondo, Nigeria. Her research interest is on biomedical image processing. She has a M.Tech in Computer Science at the Federal University of Technology, Akure, Nigeria. 